\title{
A New Fuzzy-Valued Additive Measure
}

\section{Cai-Li Zhou}

College of Mathematics and Information Science, Hebei University, Baoding, China

\section{Email address:}

pumpkinlili@163.com

\section{To cite this article:}

Cai-Li Zhou. A New Fuzzy-Valued Additive Measure. American Journal of Applied Mathematics. Vol. 3, No. 6, 2015 , pp. $259-264$. doi: $10.11648 /$ j.ajam.20150306.14

\begin{abstract}
In this paper, we firstly invoke gradual Hausdorff metric to define a new additive fuzzy-valued measure on the ordinary measurable space. Then, from the view of a fuzzy number as a crisp interval of gradual numbers, we show that the new fuzzy-valued measure can be characterized by two gradual number-valued measures. Finally, we investigate some of its properties and structural characterizations.
\end{abstract}

Keywords: Gradual Number, Fuzzy Number, Fuzzy-Valued Measure

\section{Introduction}

The concept of fuzzy set was introduced by Zadeh in 1965 . After that, many applications of fuzzy sets have been developed. One of them is the fuzzy measure. The topic of fuzzy measures has received much attention because of its usefulness in several applied fields like mathematical economics and optimal control. In fuzzy mathematics the term "fuzzy measure" is used to denote the notion which generalizes a measure - an additive function which maps $\boldsymbol{\sigma}$ - algebra to the Banach set. These generalizations can be made in two dominant directions but with one common property - they all have a grade of uncertainty. The first approach treats the fuzzy measure as a nonadditive function which maps $\boldsymbol{\sigma}_{-}$algebra to the set of reals. Additivity is usually substituted by some form of continuity. The examples of this kind of fuzzy measure are possibility, plausibility, null-measures. the second type of fuzzy measure (named fuzzy-valued measure) as a natural generalization of set-valued (or multivalued) measure is considered. Significant contributions in this area were made by Stojakovic [7], Xue, $\mathrm{Ha}$ and $\mathrm{Wu}[9]$ and $\mathrm{Wu}$, Xue and $\mathrm{Wu}[8]$, etc.

Metric on the space of fuzzy sets plays a very important role in fuzzy application systems. Recently, Zhou and Zhang [12] introduced a fuzzy metric on the space of fuzzy numbers and called it gradual Hausdorff metric. In particular, by gradual Hausdorff metric, the authors introduced a new infinite sum for sequences of fuzzy numbers and discussed its properties. In the present paper, based on the new concept of infinite sum introduced by Zhou and Zhang [12], we introduce a new additive fuzzy-valued measure. The new fuzzy-valued measure can be characterized by two gradual number-valued measures. By virtue of this property, we prove the finite additivity and monotonicity of the new measure which are similar to the classical measure and discuss its structural characterizations.

The organization of the paper is as follows. In Section 2, we state some basic results about gradual numbers, fuzzy numbers and gradual Hausdorff metrics. In Section 3, we introduce the new fuzzy-valued measure. From the view of a fuzzy number as a crisp interval of gradual numbers, we investigate some of its properties.

\section{Preliminaries}

In this section, we state some basic concepts about gradual numbers, fuzzy numbers and gradual Hausdorff metrics.

Definition 2.1. [4] A gradual number $\widetilde{r}$ is defined by an assignment function

$$
A_{\widetilde{r}}:(0,1] \rightarrow R
$$

Naturally a nonnegative gradual number is defined by its assignment function from $(0,1]$ to $[0,+\infty)$.

In the sequel, $\widetilde{r}(\alpha)$ may be substituted for $A_{\tilde{r}}(\alpha)$. The set of all gradual numbers (resp. nonnegative gradual numbers) is denoted by $R(I)$ (resp. $\left.R^{*}(I)\right)$. A crisp element $b \in R$ has its own assignment function $\tilde{b}:(0,1] \rightarrow R$ defined by $\tilde{b}(\alpha)=b$ for each $\alpha \in(0,1]$. We call such elements in $R(I)$ constant gradual numbers. In particular, $\widetilde{0}$ (resp. $\widetilde{1}$ ) 
denotes constant gradual number defined by $\widetilde{0}(\alpha)=0$ (resp. $\widetilde{1}(\alpha)=1)$ for all $\alpha \in(0,1]$.

Definition 2.2. [4] Let $\widetilde{r}, \widetilde{s} \in R(I)$. The arithmetic operations of $\widetilde{r}$ and $\widetilde{s}$ are defined as follows:

(1) $(\widetilde{r}+\widetilde{s})(\alpha)=\widetilde{r}(\alpha)+\widetilde{s}(\alpha), \forall \alpha \in(0,1]$;

(2) $(\widetilde{r}-\widetilde{s})(\alpha)=\widetilde{r}(\alpha)-\widetilde{s}(\alpha), \forall \alpha \in(0,1]$;

(3) $(\widetilde{r} \cdot \widetilde{s})(\alpha)=\widetilde{r}(\alpha) \cdot \widetilde{s}(\alpha), \forall \alpha \in(0,1]$;

(4) $\left(\frac{\widetilde{r}}{\widetilde{s}}\right)(\alpha)=\frac{\widetilde{r}(\alpha)}{\widetilde{s}(\alpha)}$, if $\widetilde{s}(\alpha) \neq 0, \forall \alpha \in(0,1]$.

Definition 2.3. [10] Let $\left\{\widetilde{r}_{n}\right\}_{n \in N} \subseteq R(I)$ and $\widetilde{r} \in R(I)$.

(1) $\left\{\widetilde{r}_{n}\right\}_{n \in N}$ is said to converge to $\widetilde{r}$ if for each $\alpha \in(0,1]$, $\lim _{n \rightarrow \infty} \widetilde{r}_{n}(\alpha)=\widetilde{r}(\alpha)$ and it is denoted as $\lim _{n \rightarrow \infty} \widetilde{r}_{n}=\widetilde{r}$.

(2) If $\lim _{n \rightarrow \infty} \sum_{i=1}^{n} \widetilde{r}_{i}$ exists, then the infinite sum of sequence $\left\{\widetilde{r}_{n}\right\}_{n \in N}$ is defined by

$$
\sum_{n=1}^{\infty} \widetilde{r}_{n}=\lim _{n \rightarrow \infty} \sum_{i=1}^{n} \widetilde{r}_{i}
$$

In the following, we describe some basic results about fuzzy numbers. A fuzzy number is a normal, convex, upper semicontinuous and compactly supported fuzzy set on $R$. In the sequel, let $F_{c}(R)$ denote the family of all fuzzy numbers. According to Fortin and Dubois and Fargier [4], a fuzzy number $\tilde{A}$ can be viewed as a particular gradual interval $\widetilde{A}=\left[\widetilde{a}^{-}, \widetilde{a}^{+}\right]$, where $\widetilde{a}^{-}$and $\widetilde{a}^{+}$are defined by

$$
\tilde{a}^{-}(\alpha)=\inf \{x: \widetilde{A}(x) \geq \alpha\}
$$

and

$$
\widetilde{a}^{+}(\alpha)=\sup \{x: \widetilde{A}(x) \geq \alpha\}
$$

for each $\alpha \in(0,1]$, respectively.

A crisp interval $A=\left[a^{-}, a^{+}\right]$can be regarded as a degenerate fuzzy number bounded by two constant gradual numbers and a gradual number $\widetilde{r}$ as a degenerate fuzzy number $\{\widetilde{r}\}$. We call that $\widetilde{A}$ is a nonnegative fuzzy number if $\widetilde{a}^{-}(\alpha) \geq 0$ for each $\alpha \in(0,1]$. Let $F_{c}^{*}(R)$ denote the set of all nonnegative fuzzy numbers. Note that the boundaries of conventional intervals are real numbers, the boundaries of fuzzy numbers are gradual numbers. Thus, in the same way as defining crisp interval, we can define relation, sum and scalar multiplication on the space of fuzzy numbers as follows:

Let $\widetilde{A}=\left[\widetilde{a}^{-}, \widetilde{a}^{+}\right]$and $\widetilde{B}=\left[\tilde{b}^{-}, \widetilde{b}^{+}\right]$be in $F_{c}(R)$ and $\gamma \in R$. Define

(1) $\widetilde{A}=\widetilde{B}$ if and only if $\widetilde{a}^{-}=\widetilde{b}^{-}$and $\widetilde{a}^{+}=\widetilde{b}^{+}$;

(2) $\widetilde{A} \leq \widetilde{B}$ if and only if $\widetilde{a}^{-} \leq \widetilde{b}^{-}$and $\widetilde{a}^{+} \leq \widetilde{b}^{+}$;

(3) $\widetilde{A} \oplus \widetilde{B}=\left[\widetilde{a}^{-}+\widetilde{b}^{-}, \widetilde{a}^{+}+\widetilde{b}^{+}\right]$;
(4) $\widetilde{\gamma A}=\left[\widetilde{\gamma a}^{-}, \widetilde{\alpha}^{+}\right]$if $\gamma \geq 0$ and $\widetilde{\gamma A}=\left[\widetilde{\gamma a}^{+}, \widetilde{\gamma}^{-}\right]$if $\gamma<0$.

Definition 2.4. [12] Let $\widetilde{A}=\left[\widetilde{a}^{-}, \widetilde{a}^{+}\right]$and $\widetilde{B}=\left[\widetilde{b}^{-}, \widetilde{b}^{+}\right]$ be in $F_{c}(R)$. Define

$$
\widetilde{d}_{H}(\widetilde{A}, \widetilde{B})=\max \left\{\left|\widetilde{a}^{-}-\widetilde{b}^{-}\right|,\left|\widetilde{a}^{+}-\widetilde{b}^{+}\right|\right\} .
$$

We call $\widetilde{d}_{H}$ gradual Hausdorff metric on $F_{c}(R)$. In particular, we define

$$
\|\widetilde{A}\|=\widetilde{d}_{H}(\tilde{A}, \hat{0})
$$

where $\hat{0}$ is the fuzzy number $\{\widetilde{0}\}$.

Definition 2.5 [12] Let $\left\{\widetilde{A}_{n}\right\}_{n \in N} \subseteq F_{c}(R)$ and $\tilde{A} \in F_{c}(R)$. We call that $\left\{\widetilde{A}_{n}\right\}_{n \in N}$ converges to $\widetilde{A}$ with respect to the gradual Hausdorff metric $\widetilde{d}_{H}$ if and only if

$$
\lim _{n \rightarrow \infty} \widetilde{d}_{H}\left(\tilde{A}_{n}, \widetilde{A}\right)=\tilde{0} .
$$

We denote it by $\lim _{n \rightarrow \infty} \widetilde{A}_{n}=\tilde{A}$ or $\tilde{A}_{n} \stackrel{\tilde{d}_{H}}{\longrightarrow} \tilde{A}$.

Definition 2.6 [12] Let $\left\{\tilde{A}_{n}\right\}_{n \in N} \subseteq F_{c}(R)$ and $\widetilde{S}_{n}=\oplus_{i=1}^{n} \widetilde{A}_{i}$ the partial sum of sequence $\left\{\widetilde{A}_{n}\right\}_{n \in N}$. If the sequence $\left\{\widetilde{S}_{n}\right\}_{n \in N}$ converges with respect to $\widetilde{d}_{H}$, then the infinite sum $\oplus_{n=1}^{\infty} \widetilde{A}_{n}$ of sequence $\left\{\widetilde{A}_{n}\right\}_{n \in N}$ is said to be convergent and we also write

$$
\oplus_{n=1}^{\infty} \widetilde{A}_{n}=\lim _{n \rightarrow \infty} \widetilde{S}_{n}=\lim _{n \rightarrow \infty} \oplus_{i=1}^{n} \widetilde{A}_{i}
$$

i.e., if $\oplus_{n=1}^{\infty} \widetilde{A}_{n}=\widetilde{A}$, then

$$
\lim _{n \rightarrow \infty} \widetilde{d}_{H}\left(\oplus_{i=1}^{n} \widetilde{A}_{i}, \widetilde{A}\right)=\widetilde{0} .
$$

Definition 2.7 [12] Let $\widetilde{A}=\left[\widetilde{a}^{-}, \widetilde{a}^{+}\right]$be in $F_{c}(R)$. The gradual number-valued support function $\widetilde{s}(p, \widetilde{A})$ of $\widetilde{A}$ is defined as follows:

$$
\widetilde{s}(p, \widetilde{A})=\sup \{p \widetilde{r}: \widetilde{r} \in \widetilde{A}\}, \forall p \in R .
$$

Definition 2.8 [11] Let $(X, \Sigma)$ be a measurable space. A mapping $\tilde{m}: \Sigma \rightarrow R^{*}(I)$ is called a gradual number-valued measure if it satisfies the following two conditions:

(1) $\tilde{m}(\phi)=\widetilde{0}$;

(2) if $A_{1}, A_{2} \ldots$ are in $\Sigma$, with $A_{i} \cap A_{j}=\phi$ for $i \neq j$, then 


$$
\widetilde{m}\left(\bigcup_{i=1}^{\infty} A_{i}\right)=\sum_{i=1}^{\infty} \tilde{m}\left(A_{i}\right) .
$$

The second condition is called countable additivity of the gradual number-valued measure $\tilde{m}$. We say that $(X, \Sigma, \widetilde{m})$ is a gradual number-valued measure space.

For more details on gradual numbers, gradual Hausdorff metrics and gradual number-valued measures, we refer the reader to $[1,4-6,11,12]$.

\section{Main Results}

Firstly, we introduce the new fuzzy-valued measure as follows:

Definition 3.1 Le $(X, \Sigma)$ be a measurable space. A mapping $\tilde{M}: \Sigma \rightarrow F_{c}^{*}(R)$ is called a fuzzy-valued measure if it satisfies the following two conditions:

(1) $\tilde{M}(\phi)=\hat{0}$;

(2) $\tilde{M}\left(\bigcup_{n=1}^{\infty} A_{n}\right)=\oplus_{n=1}^{\infty} \tilde{M}\left(A_{n}\right)$ exists for any sequence $\left\{A_{n}\right\}_{n \in N}$ of disjoint measurable sets.

The second condition is called countable additivity of the fuzzy-valued measure $\tilde{M}$. We say that $(X, \Sigma, \tilde{M})$ is a fuzzy-valued measure space.

Theorem 3.2 Let $(X, \Sigma)$ be a measurable space and $\widetilde{M}: \Sigma \rightarrow F_{c}^{*}(R)$ a mapping. $\tilde{M}$ is a fuzzy-valued measure if and only if $\widetilde{M}^{-}, \widetilde{M}^{+}: \Sigma \rightarrow R^{*}(I)$ defined by

$$
\tilde{M}^{-}(A)=(\tilde{M}(A))^{-} \text {and } \tilde{M}^{+}(A)=(\tilde{M}(A))^{+}
$$

are two gradual number-valued measures.

Proof. Necessity. Suppose that $\widetilde{M}: \Sigma \rightarrow F_{c}^{*}(R)$ is a fuzzy-valued measure. Then $\tilde{M}(\phi)=\hat{0}$. It follows that

$$
\tilde{M}^{-}(\phi)=(\tilde{M}(\phi))^{-}=\tilde{0} \text { and } \tilde{M}^{+}(\phi)=(\tilde{M}(\phi))^{+}=\tilde{0} \text {. If }
$$
$A_{1}, A_{2} \ldots$ are in $\Sigma$, with $A_{i} \cap A_{j}=\phi$ for $i \neq j$, then, by Theorem 3.9 [12], we have

$$
\begin{aligned}
& \tilde{M}\left(\bigcup_{n=1}^{\infty} A_{n}\right) \\
& =\oplus_{n=1}^{\infty} \tilde{M}\left(A_{n}\right) \\
& =\left[\sum_{n=1}^{\infty}\left(\tilde{M}\left(A_{n}\right)\right)^{-}, \sum_{n=1}^{\infty}\left(\tilde{M}\left(A_{n}\right)\right)^{+}\right] .
\end{aligned}
$$

This implies that

$$
\begin{aligned}
& \tilde{M}^{-}\left(\bigcup_{n=1}^{\infty} A_{n}\right) \\
& =\left[\tilde{M}\left(\bigcup_{n=1}^{\infty} A_{n}\right)\right]^{-} \\
& =\left(\oplus_{n=1}^{\infty} \tilde{M}\left(A_{n}\right)\right)^{-} \\
& =\sum_{n=1}^{\infty}\left(\tilde{M}\left(A_{n}\right)\right)^{-} \\
& =\sum_{n=1}^{\infty} \tilde{M}^{-}\left(A_{n}\right) .
\end{aligned}
$$

In the same way, we have

$$
\widetilde{M}^{+}\left(\bigcup_{n=1}^{\infty} A_{n}\right)=\sum_{n=1}^{\infty} \tilde{M}^{+}\left(A_{n}\right) .
$$

Hence, $\tilde{M}^{-}$and $\tilde{M}^{+}$are two gradual number-valued measures.

On the contrary, we prove the sufficiency. Suppose that $\tilde{M}^{-}$and $\tilde{M}^{+}$are two gradual number-valued measures. According to Definition 2.8, we have $\tilde{M}^{-}(\phi)=(\tilde{M}(\phi))^{-}=\widetilde{0}$ and $\tilde{M}^{+}(\phi)=(\tilde{M}(\phi))^{+}=\widetilde{0}$. It follows that $\tilde{M}(\phi)=\hat{0}$. If $A_{1}, A_{2} \ldots$ are in $\Sigma$, with $A_{i} \cap A_{j}=\phi$ for $i \neq j$, then we have

$$
\begin{aligned}
& \tilde{M}^{-}\left(\cup_{n=1}^{\infty} A_{n}\right) \\
& =\sum_{n=1}^{\infty} \tilde{M}^{-}\left(A_{n}\right) \\
& =\sum_{n=1}^{\infty}\left(\tilde{M}\left(A_{n}\right)\right)^{-}
\end{aligned}
$$

and

$$
\begin{aligned}
& \tilde{M}^{+}\left(\cup_{n=1}^{\infty} A_{n}\right) \\
& =\sum_{n=1}^{\infty} \tilde{M}^{+}\left(A_{n}\right) \\
& =\sum_{n=1}^{\infty}\left(\tilde{M}\left(A_{n}\right)\right)^{+} .
\end{aligned}
$$

By Theorem 3.9 [12], we have

$$
\begin{aligned}
& \tilde{M}\left(\bigcup_{n=1}^{\infty} A_{n}\right) \\
& =\left[\left(\tilde{M}\left(\bigcup_{n=1}^{\infty} A_{n}\right)\right),\left(\tilde{M}\left(\bigcup_{n=1}^{\infty} A_{n}\right)\right)^{+}\right] \\
& =\left[\tilde{M}^{-}\left(\bigcup_{n=1}^{\infty} A_{n}\right), \tilde{M}^{+}\left(\bigcup_{n=1}^{\infty} A_{n}\right)\right] \\
& =\left[\sum_{n=1}^{\infty}\left(\tilde{M}\left(A_{n}\right)\right)^{-}, \sum_{n=1}^{\infty}\left(\tilde{M}\left(A_{n}\right)\right)^{+}\right] \\
& =\oplus_{n=1}^{\infty}\left[\left(\tilde{M}\left(A_{n}\right)\right)^{-},\left(\tilde{M}\left(A_{n}\right)\right)^{+}\right] \\
& =\oplus_{n=1}^{\infty} \tilde{M}\left(A_{n}\right) .
\end{aligned}
$$

Hence, $\tilde{M}$ is a fuzzy-valued measure. This completes the proof. 
Theorem 3.2 shows that a fuzzy-valued measure $\tilde{M}$ can be characterized by two gradual number-valued measures $\widetilde{M}^{-}$ and $\tilde{M}^{+}$. Hence, in the sequel, we denote $\tilde{M}$ by $\left[\widetilde{M}^{-}, \tilde{M}^{+}\right]$.

Theorem 3.3 Let $(X, \Sigma, \tilde{M})$ be a fuzzy-valued measure space and $A, B \in \Sigma$.

(1) If $A \cap B=\phi$, then

$$
\tilde{M}(A \cup B)=\tilde{M}(A)+\tilde{M}(B)
$$

(2) If $A \subseteq B$, then $\tilde{M}(A) \leq \tilde{M}(B)$.

(3)If $A \cap B \neq \phi$, then

$$
\tilde{M}(A \cup B) \leq \tilde{M}(A)+\tilde{M}(B) .
$$

Proof. (1) Suppose that $A \cap B=\phi$. Then, by Theorem 3.2 and properties of gradual number-valued measures, we have

$$
\tilde{M}^{-}(A \cup B)=\tilde{M}^{-}(A)+\tilde{M}^{-}(B)
$$

and

$$
\widetilde{M}^{+}(A \cup B)=\widetilde{M}^{+}(A)+\widetilde{M}^{+}(B)
$$

It follows that

$$
\begin{aligned}
& \tilde{M}(A \cup B) \\
& =\left[\tilde{M}^{-}(A \cup B), \tilde{M}^{+}(A \cup B)\right] \\
& =\left[\tilde{M}^{-}(A)+\tilde{M}^{-}(B), \tilde{M}^{+}(A)+\tilde{M}^{+}(B)\right] \\
& =\left[\tilde{M}^{-}(A), \tilde{M}^{+}(A)\right]+\left[\tilde{M}^{-}(B)+\tilde{M}^{+}(B)\right] \\
& =\tilde{M}(A)+\tilde{M}(B) .
\end{aligned}
$$

(2) If $A \subseteq B$, then, by Theorem 3.4 [11], we have $\tilde{M}^{-}(A) \leq \tilde{M}^{-}(B)$ and $\tilde{M}^{+}(A) \leq \tilde{M}^{+}(B)$. It follows that $\tilde{M}(A) \leq \tilde{M}(B)$.

(3) If $A \cap B \neq \phi$, then we have $A \cup B=A \cup(B \backslash A)$ and $A \cap(B \backslash A)=\phi$. Thus, according to conclusion (1), we have

$$
\tilde{M}(A \cup B)=\tilde{M}(A)+\tilde{M}(B \backslash A) .
$$

According to conclusion (2), we have $\tilde{M}(B \backslash A) \leq \tilde{M}(B) . \quad$ It follows that $\tilde{M}(A \cup B) \leq \tilde{M}(A)+\tilde{M}(B)$. This completes the proof.

Theorem 3.4 Suppose that $\tilde{M}: \Sigma \rightarrow F_{c}^{*}(R)$ is a fuzzy-valued measure on the measurable space $(X, \Sigma)$, then

$$
\widetilde{v}_{p}(A)=\widetilde{s}(p, \tilde{M}(A)), \forall A \in \Sigma
$$

is a gradual number-valued measure.

Proof. Obviously,

$$
\widetilde{v}_{p}(\phi)=\widetilde{s}(p, \tilde{M}(\phi))=\widetilde{0}
$$

If $A_{1}, A_{2} \ldots$ are in $\Sigma$, with $A_{i} \cap A_{j}=\phi$ for $i \neq j$, then $\tilde{M}\left(\cup_{n=1}^{\infty} A_{n}\right)=\oplus_{n=1}^{\infty} \tilde{M}\left(A_{n}\right)$. By Theorem 3.11 [12], we have

$$
\begin{aligned}
& \widetilde{v}_{p}\left(\cup_{n=1}^{\infty} A_{n}\right) \\
& =\widetilde{s}\left(p, \tilde{M}\left(\cup_{n=1}^{\infty} A_{n}\right)\right) \\
& =\widetilde{s}\left(p, \oplus_{n=1}^{\infty} \tilde{M}\left(A_{n}\right)\right) \\
& =\sum_{n=1}^{\infty} \widetilde{s}\left(p, \tilde{M}\left(A_{n}\right)\right) \\
& =\sum_{n=1}^{\infty} \widetilde{v}_{p}\left(A_{n}\right),
\end{aligned}
$$

which implies that $\widetilde{v}_{p}$ is a gradual number-valued measure. This completes the proof.

If $\tilde{M}: \Sigma \rightarrow F_{c}^{*}(R)$ is a fuzzy-valued measure on $(X, \Sigma)$, for fixed $x \in\{-1,1\}$, define

$$
\left|\widetilde{v}_{x}\right|(A)=|\widetilde{s}(x, \tilde{M}(A))|, \forall A \in \Sigma
$$

then $\left|\widetilde{\boldsymbol{v}}_{1}\right|$ and $\left|\widetilde{\boldsymbol{v}}_{-1}\right|$ are two gradual number-valued measures. We call that

$$
\widetilde{v}(A)=\left|\widetilde{v}_{1}\right|(A)+\left|\widetilde{v}_{-1}\right|(A)
$$

is a gradual number-valued controlling measure of $\tilde{M}$.

Theorem 3.5 Let $\tilde{M}: \Sigma \rightarrow F_{c}^{*}(R)$ be a fuzzy-valued measure on measurable space $(X, \Sigma)$. Then we have

$$
\|\tilde{M}(A)\| \leq \widetilde{v}(A) \leq \widetilde{v}(X), \forall A \in \Sigma
$$

Proof. Firstly, we prove that $\|\tilde{M}(A)\| \leq \tilde{v}(A)$. By Definition 2.4 and Definition 3.1, we have

$$
\begin{aligned}
& \|\tilde{M}(A)\| \\
& =\widetilde{d}_{H}(\tilde{M}(A), \hat{0}) \\
& =\max \left\{\left|\tilde{M}^{-}(A)\right|,\left|\widetilde{M}^{+}(A)\right|\right\} \\
& =\max \left\{\left|\widetilde{v}_{-1}\right|(A),\left|\widetilde{v}_{1}\right|(A)\right\} \\
& \leq\left|\widetilde{v}_{-1}\right|(A)+\left|\widetilde{v}_{1}\right|(A) \\
& =\widetilde{v}(A) .
\end{aligned}
$$


Secondly, since $A \subseteq X$, by Theorem 3.4 [11], it is easy to see that $\widetilde{v}(A) \leq \widetilde{v}(X)$. This completes the proof.

Theorem 3.6 Let $(X, \Sigma)$ be a measurable space and $\widetilde{M}: \Sigma \rightarrow F_{c}^{*}(R)$ a fuzzy-valued measure on $(X, \Sigma)$. If $A$ and $A_{n} \quad(n \in N)$ are in $\Sigma$ such that $A_{n} \uparrow A$, then

$$
\lim _{n \rightarrow \infty} \widetilde{d}_{H}\left(\tilde{M}\left(A_{n}\right), \tilde{M}(A)\right)=\widetilde{0} .
$$

Proof. If $A_{n} \uparrow A$, then for any $n \in N$, we have

$$
\tilde{M}(A)=\tilde{M}\left(A_{n}\right) \oplus \tilde{M}\left(A \backslash A_{n}\right) .
$$

According to Theorem 3.3 [12] and Theorem 3.5, we have

$$
\begin{aligned}
& \tilde{d}_{H}\left(\tilde{M}\left(A_{n}\right), \tilde{M}(A)\right) \\
& =\widetilde{d}_{H}\left(\tilde{M}\left(A_{n}\right) \oplus \hat{0}, \tilde{M}\left(A_{n}\right) \oplus \tilde{M}\left(A \backslash A_{n}\right)\right) \\
& \leq \widetilde{d}_{H}\left(\tilde{M}\left(A_{n}\right), \tilde{M}\left(A_{n}\right)\right)+\tilde{d}_{H}\left(\hat{0}, \tilde{M}\left(A \backslash A_{n}\right)\right) \\
& =\left\|\tilde{M}\left(A \backslash A_{n}\right)\right\| \\
& \leq \tilde{v}\left(A \backslash A_{n}\right) \rightarrow \tilde{0}
\end{aligned}
$$

as $n \rightarrow \infty$. This completes the proof.

Definition 3.7 Let $(X, \Sigma)$ be a gradual number-valued measure space and $\tilde{M}: \Sigma \rightarrow F_{c}^{*}(R)$ a fuzzy-valued measure on $(X, \Sigma)$. We call that $\tilde{M}$ is continuous absolutely with respect to $\widetilde{m}$ if for any $A \in \Sigma, \widetilde{m}(A)=\widetilde{0}$, then $\tilde{M}(A)=\hat{0}$, denoted as $\tilde{M}<\widetilde{m}$. We call that $\tilde{M}$ is singular with respect to $\tilde{m}$ if there exist $N \in \Sigma$ such that for any $A \in \Sigma, \tilde{M}\left(A \cap N^{c}\right)=\hat{0}$, denoted as $\tilde{M} \perp \widetilde{m}$.

Theorem 3.8 Let $(X, \Sigma, \tilde{M})$ be a fuzzy-valued measure space. Then $\tilde{M}$ is continuous absolutely with respect to its gradual controlling measure $\widetilde{v}$.

Proof. In fact, from definition of gradual controlling measure, we have

$$
\begin{aligned}
& \tilde{v}(A) \\
& =\left|\widetilde{v}_{1}\right|(A)+\left|\widetilde{v}_{-1}\right|(A) \\
& =\tilde{M}^{-}(A)+\tilde{M}^{+}(A)
\end{aligned}
$$

for each $A \in \Sigma$. Hence, for any $A \in \Sigma$, if $\widetilde{v}(A)=\widetilde{0}$, then $\tilde{M}^{-}(A)+\tilde{M}^{+}(A)=\widetilde{0}$. It follows that $\widetilde{M}^{-}(A)=\widetilde{M}^{+}(A)=\widetilde{0}$, which implies that

$$
\tilde{M}(A)=\left[\tilde{M}^{-}(A), \tilde{M}^{+}(A)\right]=\hat{0} .
$$

Thus, $\widetilde{M}<<\widetilde{v}$. This completes the proof.

Theorem 3.9 Let $(X, \Sigma, \tilde{m})$ be a gradual number-valued measure space and $\tilde{M}$ a fuzzy-valued measure on $(X, \Sigma)$. Then

(1) $\tilde{M}<<\tilde{m}$ if and only if $\widetilde{v}<<\tilde{m}$;

(2) $\tilde{M} \perp \widetilde{m}$ if and only if $\widetilde{v} \perp \tilde{m}$;

(3) $\tilde{M}<<\tilde{m}$ and $\tilde{M} \perp \tilde{m}$ if and only if $\tilde{M} \equiv \hat{0}$.

Proof. (1) Suppose that $\tilde{M}<<\tilde{m}$. For any $A \in \Sigma$, if $\tilde{m}(A)=\widetilde{0}$, then we have $\tilde{M}(A)=\hat{0}$. It follows that

$$
\widetilde{v}(A)=\widetilde{M}^{-}(A)+\widetilde{M}^{+}(A)=\widetilde{0} .
$$

From Theorem 3.8, sufficiency is obvious.

(2) If $\widetilde{M}$ is singular with respect to $\widetilde{m}$, then there exists $N \in \Sigma$ such that for any $A \in \Sigma, \tilde{M}\left(A \cap N^{c}\right)=\hat{0}$. It follows that $\tilde{M}^{-}\left(A \cap N^{c}\right)=\widetilde{0}$ and $\tilde{M}^{+}\left(A \cap N^{c}\right)=\widetilde{0}$. Hence $\widetilde{v}\left(A \cap N^{c}\right)=\widetilde{0}$,i.e., $\widetilde{v} \perp \widetilde{m}$.

Conversely, suppose that $\widetilde{v}$ is singular with respect to $\tilde{m}$. Then there exists $N \in \Sigma$ such that for any $A \in \Sigma$,we have $\widetilde{v}\left(A \cap N^{c}\right)=\widetilde{0}$.It follows that $\tilde{M}^{-}\left(A \cap N^{c}\right)=\widetilde{0}$ and $\widetilde{M}^{+}\left(A \cap N^{c}\right)=\tilde{0}$. Hence $\tilde{M}\left(A \cap N^{c}\right)=\hat{0}$.

(3) If $\tilde{M}<<\tilde{m}$ and $\tilde{M} \perp \tilde{m}$, then $\widetilde{v}<<\tilde{m}$ and $\widetilde{v} \perp \tilde{m}$. It follows that $\widetilde{V} \equiv \widetilde{0}$, which implies that $\tilde{M} \equiv \hat{0}$. If $\widetilde{M} \equiv \hat{0}$, then $\widetilde{v} \equiv \widetilde{0}$. It follows that $\widetilde{v}<<\widetilde{m}$ and $\widetilde{v} \perp \tilde{m}$. By means of (1) and (2), we have $\tilde{M}<<\tilde{m}$ and $\tilde{M} \perp \tilde{m}$. This completes the proof.

\section{Conclusions}

In the current paper, we introduce a new fuzzy-valued measure and discuss some of its properties. In all applications which involve measure, when measurement or data are fuzzy, the measure defined in this paper can be applied. The directions of further investigation are numerous: specific properties of this new fuzzy-valued measure, integral with respect to the measure, expectation and conditional expectation, and application in economy.

\section{Acknowledgements}

The project is supported by the Youth Scientific Research Foundation of Education Department of Hebei Province (QN2015005, QN2015026), National Natural Science Foundation of China (41201327), Natural Science Foundation of Hebei Province (A2013201119) and Intramural Personnel Training Scientific Foundation of Hebei University. Their financial support is gratefully acknowledged.

\section{References}

[1] D. Dubois and H. Prade, Gradual elements in a fuzzy set, Soft Computing, 12 (2008), 165 - 175. 
[2] D. Dubois, J. Fortin and P. Zielinnski, Interval PERT and its fuzzy extension, Studies in Fuzziness and Soft Computing, 252 (2010), $171-199$.

[3] J. Fortin and D. Dubois, Solving fuzzy PERT using gradual real numbers, In L. Penserini, A. Perini, and P. Peppas, editors, STAIRS 2006: Proceedings of the Third Starting AI Researcher's Symposium, IOS Press, Fairfax, VA, 160 (2006), 196 - 207.

[4] J. Fortin, D. Dubois and H. Fargier, Gradual numbers and their application to fuzzy interval analysis, IEEE Transactions on fuzzy systems, 16 (2008), 388 - 402.

[5] A. Kasperski, P. Zielinski, Using gradual numbers for solving fuzzy-valued combinatorial optimization problems, Foundations of Fuzzy Logic and Soft Computing, (2007), 656 665 .

[6] E.A. Stock, Gradual numbers and fuzzy optimization, ph.D. Thesis, University of Colorado Denver, Denver, America. 2010.
[7] M. Stojakovic, Fuzzy valued measure, Fuzzy Sets and Systems, 65 (1994), 95 - 104.

[8] J. Wu, X. Xue and C. Wu, Radon-Nikodym theorem and Vitali-Hahn-Saks theorem on fuzzy number measures in Banach spacers, Fuzzy Sets and Systems, 117 (2001) 339 - 346.

[9] X. Xue, M. Ha and C. Wu, On the extension of the fuzzy number measures in Banach spaces: Part I. Representation of the fuzzy number measures, Fuzzy Sets and Systems, 78 (1996) $347-354$.

[10] C. Zhou and P. Wang, New fuzzy probability spaces and fuzzy random variables based on gradual numbers, In: 2014 9th International Conference, BIC-TA 2014, (2014), 633 - 643.

[11] C. Zhou and J. Li, New Fuzzy Measure Based on Gradual Numbers, International Journal of Mathematical Analysis, 9 (2015), $101-110$.

[12] C. Zhou and G. Zhang, A Fuzzy Metric on the Space of Fuzzy Sets, International Journal of Mathematical Analysis, 9 (2015), 237 - 247. 